\section{Exposure to statins in early old age but not in late old age may be associated with a lower risk of developing Alzheimer's disease}

\section{QUESTION}

Question: Do statins reduce the risk of Alzheimer's disease $(\mathrm{AD})$, and is this influenced by age or presence of apolipoprotein $\mathrm{E}(\mathrm{APOE}) \varepsilon 4$ allele?

Population: 3392 members of a health maintenance organisation, aged 65 or older and without dementia.

Setting: Community based (Seattle-area, USA).

Prognostic factors: Statin use (simvastatin, lovastatin, pravastatin and atorvastatin); duration of statin use (from date of first statin prescription to date of last); APOE genotype.

Outcomes: Probable AD assessed every 2 years (screening on Cognitive Abilities Screening Instrument). Those screen positive at assessment underwent diagnostic interview using National Institute of Neurological and Communicative Disorders and Stroke - Alzheimer Disease and Related Disorders Association criteria for AD dementia. Participants who had other types of dementia were censored in analyses at the time of estimated onset.

\section{METHODS}

Design: Prospective cohort study.

Follow-up period: Average of 6.1 years.

\section{MAIN RESULTS}

Of the 3392 enrolled participants, 3099 had at least one follow-up examination and were included in the analysis. $711 \mathrm{had}$ taken statins for an average of $5.4( \pm 3.6)$ years. Self-reported comorbid vascular conditions, cigarette smoking, higher serum total cholesterol, lower high-density lipoprotein cholesterol and higher body mass index were more common in those exposed to statins. During follow-up, 263 participants developed probable $\mathrm{AD}$; mean age of onset was $82.9( \pm 5.1)$ years. Overall, statin use reduced the risk of probable $\mathrm{AD}$ (HR 0.62, 95\% CI 0.40 to $0.97)$; this analysis was adjusted for demographic characteristics and vascular risk factors. When stratified by age, the effects of statins were significant only in patients aged $65-80$ years (HR $0.44,95 \%$ CI 0.25 to 0.78 ) and not in those older than 80 years (HR $1.22,95 \%$ CI 0.61 to 2.42 ). In those who had genotype data available, statin use reduced risk of $\mathrm{AD}$ in those with an $\varepsilon 4$ allele but not in those without (HR 0.42, 95\% CI 0.20 to 0.91 vs $0.77,95 \%$ CI 0.42 to 1.40$)$.

\section{CONCLUSIONS}

Statin use in people aged 65-80 years appears to reduce the risk of $\mathrm{AD}$; however, this association between statin use and $\mathrm{AD}$ was not observed in statin users who were older than 80 at study entry.

\section{ABSTRACTED FROM}

Li G, Shofer JB, Rhew IC, et al. Age-varying association between statin use and incident Alzheimer's disease. J Am Geriatr Soc 2010;58:1311-7.

Correspondence to: $\operatorname{Dr}$ Ge Li, Mail Code s116 6EMIRECC VA Puget Sound Health Care System, 1660 South Columbian Way, Seattle WA 98108-1597, USA; gli@u.washington.edu

Sources of funding: The National Institute of Aging, the Department of Veterans affairs, the Friends of Alzheimer's Research.
$[$ a pidemiologic observations have reported an association between statin therapy and decreased risk of dementia and Alzheimer's disease (AD). The mechanisms are not fully elucidated but may include effects on vascular risk factors or direct effects on amyloid metabolism itself. However, these observations of protective effects have not been borne out in two randomised controlled trials with dementia prevention as an outcome. ${ }^{1}$ The association between statin therapy and dementia/AD risk may vary by age: in the Canadian Study of Health and Aging (CSHA), the use of statins or another lipid-lowering agent was associated with lower $\mathrm{OR}$ for $\mathrm{AD}$ only in participants $<80$ years (OR, $0.26 ; 95 \% \mathrm{Cl}, 0.09$ to 0.72 ) but not in participants $>80$ years.

This finding led the authors to re-examine these questions in a rigorously studied cohort of older persons (Adult Changes in Thought) who are members of a health maintenance organisation (Group
Health Cooperative, GHC). Three thousand and ninety-nine GHC members $\geq 65$ years of age were recruited in two cohorts and followed for a mean of 6.1 years. Statin use was associated with lower odds of $A D$ in participants $\leq 80$ years but not in participants $>80$ years.

Together with the CSHA results, these results suggest a possible protective effect of statins against $A D$ in persons $\leq 80$ years. The results are plausible mechanistically, since there is increasing evidence that the preclinical stage of $A D$ starts years before clinical symptoms and that $A D$ neurotoxic mechanisms are stage-specific. Thus it is reasonable that the protective effect of a medication might only be observed when started as early as possible in the preclinical stage of $A D$. The time may be upon us to undertake a prevention trial of statins in 'young-old' participants, very possibly with a short-term biomarker outcome as well as long- term dementia outcomes. One cannot recommend the use of statins clinically for $A D$ prevention at this time, without the results of a randomised controlled trial, since epidemiologic observations in this area have often not been borne out by trials (as in the case of estrogens).

\section{Paul B Rosenberg}

Johns Hopkins School of Medicine, Baltimore, Maryland USA

Conflicting interests PBR has received research support from Pfizer, Lilly, Merck, Elan, Janssen, National Institute of Aging and the American Foundation for Aging Research.

\section{REFERENCE}

1. McGuinness B, Craig D, Bullock R, et al. Statins for the prevention of dementia. Cochrane Database Syst Rev 2009;15:CD003160. 